\title{
Quadratic thermal terms in the deconfined phase from holography
}

\author{
Fen Zuo ${ }^{a}$ and $\mathrm{Yi}$-Hong $\mathbf{G a o}^{b}$ \\ ${ }^{a}$ School of Physics, Huazhong University of Science and Technology, \\ Wuhan 430074, China \\ ${ }^{b}$ State Key Laboratory of Theoretical Physics, Institute of Theoretical Physics, \\ Chinese Academy of Sciences, \\ P.O. Box 2735, Beijing 100190, China \\ E-mail: zuofen@hust.edu.cn, gaoyh@itp.ac.cn
}

ABSTRACT: Recent lattice simulation has uncovered many interesting properties of $\mathrm{SU}(N)$ gauge theory at finite temperature. Especially, above the deconfinement phase transition all the thermodynamic quantities acquire significant quadratic contributions in inverse temperature. Such a term is also found to dominate the logarithm of the renormalized Polyakov loop. Using the Hawking-Page transition in Anti-de Sitter space as an example, we show how such contributions can be naturally generated in the holographic approach.

Keywords: Confinement, Wilson, 't Hooft and Polyakov loops, AdS-CFT Correspondence, Black Holes

ARXIV EPRINT: 1403.2241 


\section{Contents}

1 Introduction $\quad 1$

2 The black holes in Anti-de Sitter space-time 2

3 Thermodynamics and the trace anomaly 4

4 Renormalized Polyakov loop $\quad 8$

$\begin{array}{lll}5 & \text { Results for lower spacetime dimensions } & 10\end{array}$

$\begin{array}{llr}6 & \text { Discussion } & 11\end{array}$

\section{Introduction}

Color confinement in $\mathrm{SU}(N)$ gauge theory is believed to be due to the linearly-increasing potential between colored objects when they are separated. Such distance dependence has been confirmed by lattice data. At short distances, the linear potential appears as a quadratic correction to the lead Coulomb term [1]. Such a correction could be imitated through a tachyonic gluon mass [2]. However, the theoretic origin is not clear because no dimension-2 gauge invariant operator exists. The linear potential leads to the area law of Wilson loop expectation value. In the gauge/string duality [3-5], the Wilson loop expectation value can be approximately evaluated from the minimum area of the corresponding string worldsheet $[6,7]$. Area law for the Wilson loop can be realized when the bulk spacetime ends before the worldsheet goes into the deep infrared region [8]. Along this line, the relation between linear potential and quadratic corrections in the two point correlation functions is further studied in $[9,10]$.

Recent lattice data has uncovered some similar phenomena in the deconfining phase of $\mathrm{SU}(N)$ gauge theory. It is pointed out in [11] that early lattice data for the trace anomaly in $\mathrm{SU}(3)$ gauge theory [12], scaled by $T^{4}$, is dominated by a quadratic term in inverse temperature. With an additional quartic term, the data can be fitted extremely well. Such a behavior can be made manifest when the trace anomaly is plotted scaled by $T^{2}$ [13]. The result is almost constant in the temperature region $T_{c} \lesssim T \lesssim 4 T_{c}$. Similar as the quark potential, the thermal quadratic term could also be induced from the presence of massive gluons [11] (see also [14]). Compared to the quartic term related to the bag constant, the quadratic term is suggested to reflect the bag thickness in the relevant temperature region [13]. The corresponding model with both of them, and even higher power terms, is then called "Fuzzy Bags". Such a behavior is further confirmed for pure $\mathrm{SU}(N)$ gauge theory with various $N$ [15], and suggested to hold even in the large- $N$ limit. 
Another observation concerns the behavior of the Polyakov loop above phase transition. At finite temperature, the Polyakov loop attains nonzero value in the deconfining phase, and serves as the order parameter of deconfinement $[16,17]$. Interestingly, lattice results [18] for the logarithm of the Polyakov loop in $\mathrm{SU}(3)$ gauge theory is also dominated by $1 / T^{2}$ term [19]. Such a behavior is confirmed recently for SU(4) and SU(5) gauge theory [20], and the coefficient of the quadratic term has little dependence on the gauge group.

As proposed in $[5,8]$, deconfinement of $\mathrm{SU}(N)$ gauge theory in the large $N$ limit can be described as the transition between the thermal AdS phase and the black hole phase on the gravity side. A concrete example is $\mathcal{N}=4$ super Yang-Mills theory at finite temperature on $S^{1} \times S^{3}$. Here we show explicitly that in such a theory, all the thermodynamic quantities, and also the renormalized Polyakov loop, exhibit very similar temperature behavior as those discussed above. In particular, the quadratic terms are generated consistently and make significant contributions in all of them. In [21, 22] such terms were previously studied in the so-called soft-wall models [23, 24]. However, the thermodynamic quantities and the Polyakov loop are obtained separately from different backgrounds. An interesting investigation of the thermal power contributions in arbitrary spacetime dimension is recently performed in the gravity-dilaton system [25]. In the work it is noticed that the power of the dominant term could be related to the order of the deconfinement transition and the scaling of hadron spectra. However, it seems impossible to generate simultaneously the correct powers in both the thermodynamic quantities and the Polyakov loop [26]. Also in the effective matrix model [27], it is difficult to reproduce the shape of the Polyakov loop when the thermodynamic quantities are well fitted.

The paper is organized in the following way. In the next section we review the pure thermal solutions and the Schwarzschild black hole solutions on Anti-de Sitter (AdS) space. The thermodynamics and the Polyakov loop are studied in section III and section IV, respectively. Possible generalization to lower spacetime dimensions is considered in section $\mathrm{V}$. In the final section we give a short discussion and point out possible generalization of the work.

\section{The black holes in Anti-de Sitter space-time}

First we review the thermal AdS and AdS black hole solutions, which are first given in [28] and further studied in [8]. As in [8], we keep the derivation general for arbitrary spacetime dimension $D=d+2$. Only when interpreting the results as dual to those in the boundary gauge theory, we fix $d=3$. In such a case the gravity description is known to be dual to the maximally supersymmetric gauge theory.

The gravity action considered composes of the Einstein term and a negative cosmological constant,

$$
\mathcal{J}=-\frac{1}{16 \pi G_{d+2}} \int \mathrm{d}^{d+2} y \sqrt{g}(R-2 \Lambda)
$$

where $\Lambda=-d(d+1) /\left(2 L^{2}\right)$. As well known, the action admits AdS space solution $[8,28]$

$$
\mathrm{d} s^{2}=-\left(1+\frac{r^{2}}{L^{2}}\right) \mathrm{d} t^{2}+\left(1+\frac{r^{2}}{L^{2}}\right)^{-1} \mathrm{~d} r^{2}+r^{2} \mathrm{~d} \Omega_{d}^{2}
$$


Here $\mathrm{d} \Omega_{d}^{2}$ is the metric on the unit sphere $S^{d}$, and the time coordinate $t$ is periodically identified with period $2 \pi L$. The corresponding thermal phase is obtained in the Euclidean formalism with the substitution $\tau=\mathrm{i} t$.

The action also admits the AdS-Schwarzschild black hole solution [8, 28]

$$
\mathrm{d} s^{2}=-\left(1-\frac{\omega_{d+1} M}{r^{d-1}}+\frac{r^{2}}{L^{2}}\right) \mathrm{d} t^{2}+\left(1-\frac{\omega_{d+1} M}{r^{d-1}}+\frac{r^{2}}{L^{2}}\right)^{-1} \mathrm{~d} r^{2}+r^{2} \mathrm{~d} \Omega_{d}^{2}
$$

where

$$
\omega_{d+1}=\frac{16 \pi G_{d+2}}{\operatorname{Vol}\left(S^{d}\right) d}, \quad \operatorname{Vol}\left(S^{d}\right)=\frac{2 \pi^{(d+1) / 2}}{\Gamma((d+1) / 2)} .
$$

Later we will see $M$ is the expectation value of the energy, and thus the mass of the black hole. In the limit $M \rightarrow 0$, the black hole solution reduces to pure AdS (2.2). $M$ is related to the horizon $r_{+}$as

$$
M=\frac{1}{\omega_{d+1}}\left[r_{+}^{d-1}+\frac{r_{+}^{d+1}}{L^{2}}\right]
$$

and the Hawking temperature is given by

$$
T_{H}=\frac{(d+1) r_{+}^{2}+(d-1) L^{2}}{4 \pi L^{2} r_{+}} .
$$

The temperature has a minimum bound

$$
T_{\min }=\frac{\sqrt{d^{2}-1}}{2 \pi L},
$$

above which the black hole solution exists. As shown in $[8,28]$, the thermal AdS solution (2.2) dominates at low temperature and the black hole phase is more stable at high temperature. The phase transition between them is interpreted as deconfinement in the boundary gauge theory, which takes place at

$$
T_{c}=\frac{d}{2 \pi L}>T_{\min }
$$

Both the above solutions have the topology $S^{1} \times S^{d}$ at the boundary $r \rightarrow \infty$. With a suitable choice of the transformation function, the radiuses of $S^{1}$ and $S^{d}$ can be chosen to be $\beta_{H}=T_{H}^{-1}$ and $L$ respectively. Taking the radius of $S^{d}$ to infinity, the boundary becomes $S^{1} \times R^{d}$. Equivalently, one can choose $\beta_{H} \rightarrow 0$, or $M \rightarrow \infty$ [8]. Absorbing the divergent quantities by redefining the coordinates, one arrives at the metric

$$
\mathrm{d} s^{2}=-\frac{r^{2}}{L^{2}}\left[1-\left(\frac{r_{0}}{r}\right)^{d+1}\right] \mathrm{d} t^{2}+\left\{\frac{r^{2}}{L^{2}}\left[1-\left(\frac{r_{0}}{r}\right)^{d+1}\right]\right\}^{-1} \mathrm{~d} r^{2}+\frac{r^{2}}{L^{2}} \mathrm{~d} \mathbf{x}^{2} .
$$

Now the horizon is at $r_{0}$, with $T_{H}=\frac{d+1}{4 \pi L^{2}} r_{0}$. The corresponding thermal gas solution is simply

$$
\mathrm{d} s^{2}=-\frac{r^{2}}{L^{2}} \mathrm{~d} t^{2}+\left\{\frac{r^{2}}{L^{2}}\right\}^{-1} \mathrm{~d} r^{2}+\frac{r^{2}}{L^{2}} \mathrm{~d} \mathbf{x}^{2}
$$

As expected, the black hole phase in this case dominates at any temperature, corresponding to unconfinement of $\mathcal{N}=4$ super Yang-Mills theory on $S^{1} \times R^{3}$. 


\section{Thermodynamics and the trace anomaly}

Now let us turn to the thermodynamic quantities. First we consider the case of infinite black hole mass, when the boundary is $S^{1} \times R^{d}$. In this case no other scale than the temperature exists, so the thermodynamic quantities scale with the temperature according to their dimensions

$$
p_{0}=\frac{c}{d+1} T_{H}^{d+1}, \quad \epsilon_{0}=\frac{c d}{d+1} T_{H}^{d+1}, \quad s_{0}=c T_{H}^{d},
$$

where

$$
c=\frac{L^{d}}{4 G_{d+2}}\left(\frac{4 \pi}{d+1}\right)^{d}
$$

In particular, we have

$$
c_{S}^{2} \equiv \frac{\mathrm{d} p_{0}}{\mathrm{~d} \epsilon_{0}}=1 / d, \quad \Delta_{0} \equiv \epsilon_{0}-d p_{0}=0 .
$$

As expected, the velocity of sound takes the conformal value and the trace anomaly vanishes. These results are supposed to be valid at large 't Hooft coupling, where the gravity approximation can be trusted. For $d=3$, perturbative calculation at zero coupling gives $p_{\text {free }}=4 / 3 p_{0}$, differing from the above result by a factor of $4 / 3$ [29]. For more discussions on the thermodynamic and transport properties can be found e.g. in [30]. There are also some interesting results on non-local observables recently [31].

Now we want to see the deviations of these two relations in (3.3) when the boundary space is compact. The free energy can be derived from the action, which after subtracting that of the thermal AdS space is

$$
F=-\frac{\operatorname{Vol}\left(S^{d}\right)}{16 \pi G_{d+2}}\left[\frac{r_{+}^{d+1}}{L^{2}}-r_{+}^{d-1}\right] .
$$

Then the energy can be derived through the thermal relation

$$
E=\frac{d \cdot \operatorname{Vol}\left(S^{d}\right)}{16 \pi G_{d+2}}\left[\frac{r_{+}^{d+1}}{L^{2}}+r_{+}^{d-1}\right]=M .
$$

The entropy can in turn be obtained, which turns out to be exactly the Bekenstein-Hawking entropy of the black hole

$$
S=\frac{\operatorname{Vol}\left(S^{d}\right)}{4 G_{d+2}} r_{+}^{d}
$$

By dividing the volume of the sphere $S^{d}$ with radius $L$, one obtains the corresponding densities $f, \epsilon$ and $s$. For $d=3$, the explicit expressions are

$$
\begin{aligned}
p & =-f=\frac{N^{2}}{8 \pi^{2}} \frac{r_{+}^{4}-r_{+}^{2} L^{2}}{L^{8}} \\
\epsilon & =\frac{3 N^{2}}{8 \pi^{2}} \frac{r_{+}^{4}+r_{+}^{2} L^{2}}{L^{8}} \\
s & =\frac{N^{2}}{2 \pi} \frac{r_{+}^{3}}{L^{6}}
\end{aligned}
$$


where we have used the duality relation [3]

$$
\frac{L^{4}}{l_{s}^{4}}=4 \pi g_{s} N=g_{\mathrm{YM}}^{2} N \equiv \lambda .
$$

In the high temperature limit, one finds

$$
p \rightarrow p_{0}, \quad \epsilon \rightarrow \epsilon_{0}, \quad s \rightarrow s_{0},
$$

which simply reflects the fact that $T_{H} \rightarrow \infty$ is equivalent to making the radius of the boundary $S^{d}$ infinity.

The sphere radius introduces another scale into the system. To see the violation of conformal invariance due to this, we formally calculate the quantity

$$
\Delta \equiv \epsilon-d p
$$

This could be considered as a direct generalization of $\Delta_{0}$, even though it may not be the trace of the energy momentum tensor in the boundary theory. A simple calculation then gives

$$
\begin{aligned}
\Delta / p_{0} & =\frac{d(d+1)^{2}}{2^{d+2} \pi^{2}} \frac{1}{L^{2} T_{H}^{2}}\left[1+\sqrt{1-\frac{T_{\min }^{2}}{T_{H}^{2}}}\right]^{d-1} \\
& =\frac{d(d+1)^{2}}{9 \cdot 2^{d}} \frac{T_{c}^{2}}{T_{H}^{2}}\left[1+\sqrt{1-\frac{T_{\min }^{2}}{T_{H}^{2}}}\right]^{d-1}
\end{aligned}
$$

We will mainly focus on $d=3$. In order to compare with the lattice results, we normalize the trace anomaly to $p_{\text {free }}$ instead

$$
\Delta / p_{\text {free }}=\frac{T_{c}^{2}}{2 T_{H}^{2}}\left[1+\sqrt{1-\frac{8}{9} \frac{T_{c}^{2}}{T_{H}^{2}}}\right]^{2} .
$$

It turns out $\Delta / p_{\text {free }}$ exhibits an interesting temperature dependence, as shown in figure 1. It increases to a maximum slightly above $T_{c}$, and starts to decrease with increasing temperature. In the large temperature limit, it goes to zero. The same temperature pattern is found in lattice simulation for pure $\mathrm{SU}(N)$ gauge theory. When $N$ varies, the lattice data can be well fitted by the formula [15]

$$
\frac{\Delta}{p_{\text {free }}}=\left(1-\frac{1}{\left\{1+\exp \left[\frac{\left(T / T_{c}\right)-f_{1}}{f_{2}}\right]\right\}^{2}}\right)\left(f_{3} \frac{T_{c}^{2}}{T^{2}}+f_{4} \frac{T_{c}^{4}}{T^{4}}\right),
$$

where $p_{\text {free }}=\frac{\pi^{2}}{45}\left(N^{2}-1\right) T^{4}$ is the corresponding perturbative result at zero coupling. The parameters have smooth limits when extrapolated to large $N$. The central values of the fitted parameters at large $N$ are given by

$$
\begin{aligned}
& f_{1}=0.9918, \quad f_{2}=0.0090 \\
& f_{3}=1.768, \quad f_{4}=-0.244
\end{aligned}
$$




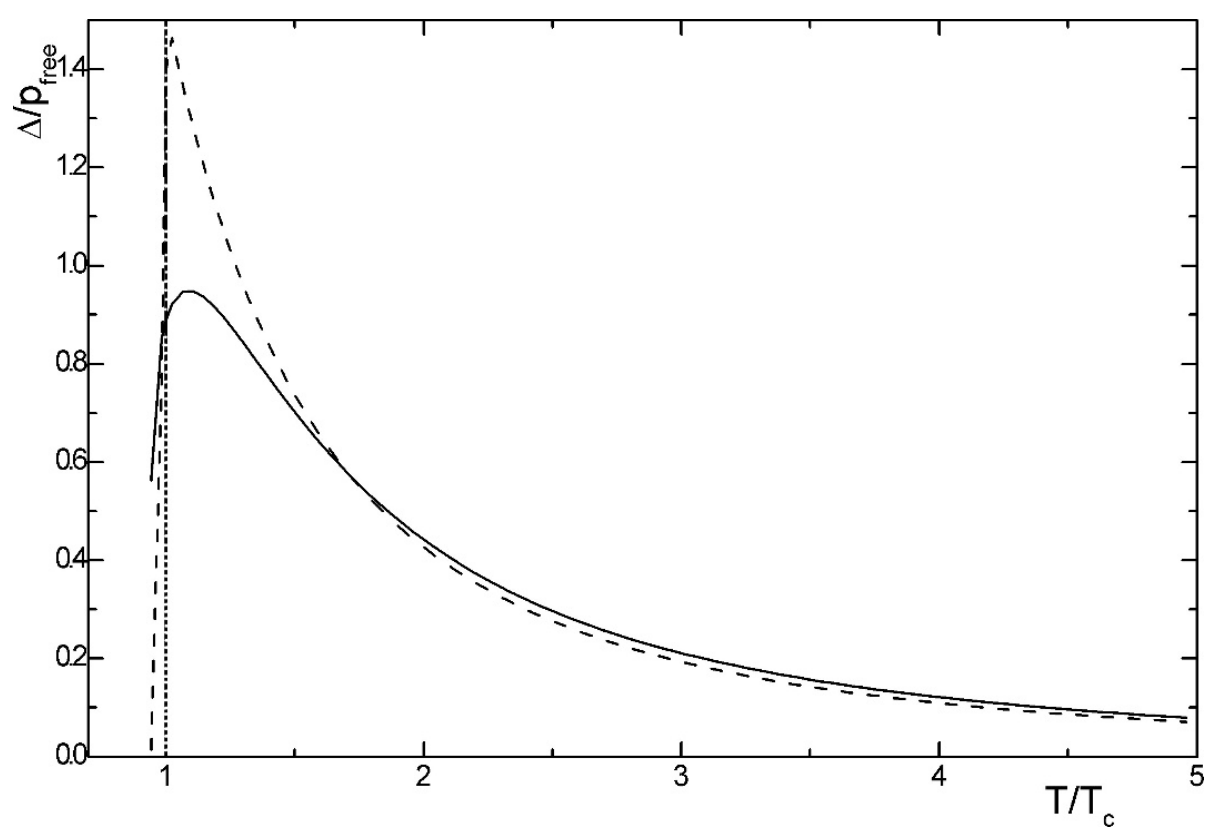

Figure 1. Holographic result (3.12) for the ratio $\Delta / p_{\text {free }}$ versus $T_{H} / T_{c}$ (solid line). Also plotted is the lattice fit (3.13) from [15] (dashed line).

In figure 1 we also plot the lattice fit (3.13) with the above parameters. Although the shape of the two curves is similar, the maximum values differ from each other. That also means, the latent heat is different. From (3.12) one reads

$$
\epsilon\left(T_{c}\right) / p_{\text {free }}\left(T_{c}\right)=8 / 9
$$

while the lattice fit gives a value around 1.4.

The appearance of the maximum in our result can be traced back to two facts. First, the temperature derivative of $\Delta$, and also $\Delta / p_{\text {free }}$, goes to $+\infty$ when the temperature approaches its minimum from above. Second, $\Delta / p_{\text {free }} \rightarrow 0^{+}$when $T_{H} \rightarrow \infty$. With these two properties, one can generally prove the existence of the maximum. Due to the quickly increasing near $T_{\min }$, the maximum is expected to appear close to $T_{\min }$. That means, close to $T_{c}$. In a similar way, one may explain the enhancement of jet quenching around phase transition, recently pointed out in a gravity-dilaton background [32]. In fact, the shape of all the physical quantities can be obtained by smoothly connecting their asymptotic form in the two limits. There will be no special structure in between, since there is no special point in the black hole solution. In particular, the phase transition is not an intrinsic property of the black hole solution. For example, one finds that the ratio $\Delta / s^{(d+1) / d}$ decreases monotonously all the way from $T_{\min }$ to infinity. Similar behavior for the bulk viscosity $\zeta$ [33-35] and the jet quenching parameter $\hat{q}$ [32], normalized in $s$, have been found in the gravity-dilaton system. This is quite different from the shear viscosity $\eta$, which takes the constant value $1 / 4 \pi$ when normalized in $s$ [36]. One may thus conjecture that any physical quantity of dimension $\alpha>0$, when normalized in $s^{\alpha / d}$, should either be a constant or decrease monotonously in the whole deconfining phase. 


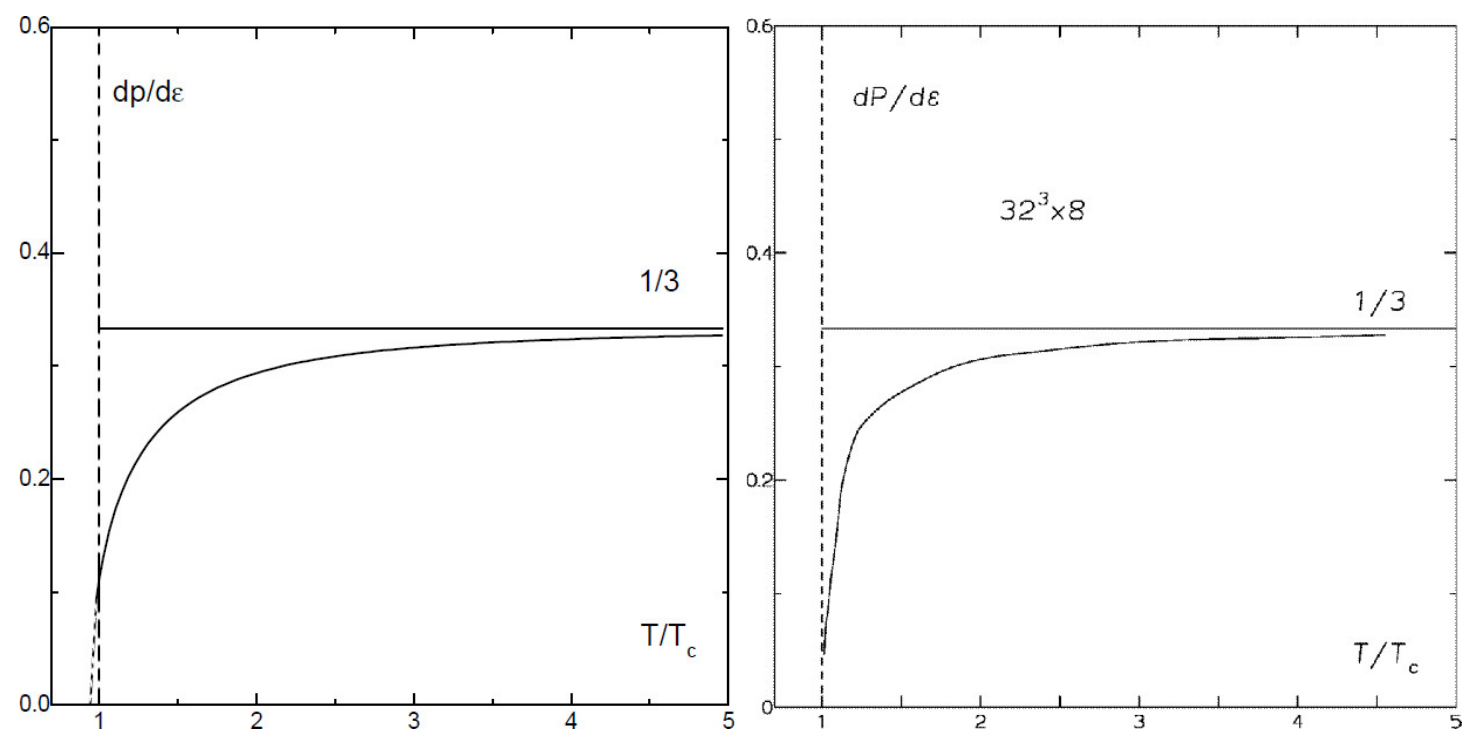

Figure 2. Holographic result for the squared speed of sound versus $T_{H} / T_{c}$ (left), in comparison with the lattice data in pure $\mathrm{SU}(3)$ gauge theory [12] (right).

In such a way we can easily sketch the behavior of the squared speed of sound. It goes to $0^{+}$when approaching $T_{\min }$ from above, and increases monotonously to its conformal value $1 / 3$ in the high temperature limit. Since $T_{c}$ is close to $T_{\min }$, the velocity would be slightly above zero at $T_{c}$. Explicitly, the squared velocity of the sound is

$$
c_{S}^{2} \equiv \frac{\mathrm{d} p}{\mathrm{~d} \epsilon}=\frac{1}{d} \sqrt{1-\frac{T_{\mathrm{min}}^{2}}{T_{H}^{2}}},
$$

and takes the value $1 / d^{2}$ at $T_{c}$. The behavior of the velocity square at $d=3$ is plotted in figure 2, which turns out to be extremely similar to the corresponding lattice result for pure SU(3) gauge theory [12]. Similar description for the thermodynamic quantities have already been constructed in the gravity-dilaton system [37-39]. It has also been extended to describe the Polyakov loop [40,41], which we will discuss in the next section.

We want to compare our result for the trace anomaly with the lattice fit in more detail. Above the phase transition, the lattice fit (3.13) simplifies due to the large exponential factor as

$$
\frac{\Delta}{p_{\text {free }}} \approx\left(f_{3} \frac{T_{c}^{2}}{T^{2}}+f_{4} \frac{T_{c}^{4}}{T^{4}}\right)
$$

Such a form was first proposed in [11]. It means that above $T_{c}$ the trace anomaly is dominated by a $T^{2}$ term, with a small constant correction. Due to the smallness of $f_{4}$, a simplified model, with only the quadratic term, is considered [13]. The corresponding pressure is then

$$
p / p_{\text {free }}=1-\frac{f_{3}}{2} \frac{T_{c}^{2}}{T^{2}}
$$

where it has been assumed $p \rightarrow p_{\text {free }}$ as $T \rightarrow \infty$. For pure glue, $p\left(T_{c}\right)$ is argued to be small, which leads to the estimation $f_{3} \simeq 2$. In general a series of terms in inverse temperature 
square can be included. The pressure then takes the form

$$
p / p_{\text {free }}=1-\frac{f_{3}}{2} \frac{T_{c}^{2}}{T^{2}}-\frac{f_{4}}{4} \frac{T_{c}^{4}}{T^{4}}+\ldots
$$

which is called the fuzzy bag model [13].

Taking the high temperature expansion of (3.12), we find

$$
\Delta / p_{\text {free }} \approx 2 \frac{T_{c}^{2}}{T_{H}^{2}}-\frac{8}{9} \frac{T_{c}^{4}}{T_{H}^{4}}
$$

Actually such a truncated expansion is almost exact above $T_{c}$. Comparing with (3.17), we immediately obtain

$$
f_{3}=2, \quad f_{4}=-\frac{8}{9} .
$$

We see indeed violation of conformal invariance is mainly due to a quadratic correction, with the coefficient $f_{3}$ close to the lattice value extrapolated to large $N$. The sign of $f_{4}$ is also the same as the lattice result for pure gauge theory, and differs from that in QCD at zero temperature and at finite temperature [42]. However, the absolute value of $f_{4}$ is about two times bigger than that from the lattice fit. Such a large negative $f_{4}$ has significant effects around the phase transition, and leads to the smallness of the latent heat.

\section{Renormalized Polyakov loop}

Now we will discuss the results for the Polyakov loop $\mathcal{L}$. In the dual description, $\mathcal{L}$ is approximately evaluated through the minimum area $S$ of the string worldsheet ending on the temporal loop $[6,7]$

$$
\mathcal{L} \approx \mathrm{e}^{-S}
$$

Due to different topologies of the solutions shown in the previous section, such a string worldsheet may exist or not [8]. The topology of the thermal AdS (2.2) is $S^{1} \times S^{d+1}$, and $R^{2} \times S^{d}$ for the AdS-Schwarzschild black hole (2.3). Due to this, in the first case no such string worldsheet exists and the corresponding Polyakov loop is vanishing. The Polyakov loop $\mathcal{L}$ is related to the quark free energy as

$$
\mathcal{L}=\mathrm{e}^{-F_{Q}(T) / T} .
$$

Thus vanishing of $\mathcal{L}$ corresponds to an infinite self energy of a single quark, and induces confinement. In the latter case, the string worldsheet that ends on the boundary $S^{1}$ simply expands the whole $R^{2}$ part. A nonzero value of the Polyakov loop is thus generated, signaling unconfinement in the black hole phase.

Now we calculate the quark free energy explicitly, through the area of the string worldsheet

$$
F_{Q}(T) \approx T S .
$$

The area is simply that of the $R^{2}$ part spanned by $t$ and $r$

$$
S_{1}=\frac{1}{2 \pi \alpha^{\prime}} \int_{0}^{\beta_{H}} \mathrm{~d} t \int_{r_{+}}^{\infty} \mathrm{d} r
$$


The integral is divergent. A finite free energy, and thus $\mathcal{L}$, can be obtained by slightly shifting the upper bound of the $r$ integral away from the boundary $r \rightarrow \infty$. This corresponds to the renormalization procedure. Here we will define the renormalized quark free energy in a different way, by subtracting the corresponding result in the limit of infinite black hole mass. As explained in the previous sections, in this case the black hole phase always dominates over the thermal AdS solution. For $d=3$ this corresponds to the fact that $\mathcal{N}=4$ Super Yang-Mills theory on the boundary $S^{1} \times R^{3}$ is always unconfining. Therefore with such a subtraction we can extract the dynamical part of the quark free energy that is related to the phase transition. Similar procedure has been employed in [22]. The corresponding worldsheet area in the large mass limit is given by

$$
S_{0}=\frac{1}{2 \pi \alpha^{\prime}} \int_{0}^{\beta_{H}} \mathrm{~d} t \int_{r_{0}}^{\infty} \mathrm{d} r
$$

Then we can define the renormalized worldsheet area as

$$
S^{R} \equiv S_{1}-S_{0}=\frac{1}{2 \pi \alpha^{\prime}} \int_{0}^{\beta_{H}} \mathrm{~d} t \int_{r_{+}}^{r_{0}} \mathrm{~d} r
$$

In [22], an arbitrary normalization constant is introduced in such an subtraction procedure, which could describe the deviation of the Polyakov loop expectation value from unity in the high temperature region. Such a deviation could be well described by the perturbative results $[20,43]$. Since we do not take into account the perturbative contributions, we simply set the constant to be zero here. The renormalized quark free energy can then be obtained as

$$
\begin{aligned}
F_{Q}^{R} & \approx T_{H} S^{R} \\
& =\frac{\sqrt{\lambda}}{d+1}\left[T_{H}-\left(T_{H}^{2}-T_{\min }^{2}\right)^{1 / 2}\right] .
\end{aligned}
$$

As a result, the logarithm of the Polyakov loop is given by

$$
\begin{aligned}
-2 \log \mathcal{L}^{R} & =2 F_{Q}^{R}\left(T_{H}\right) / T_{H} \\
& \approx \frac{2 \sqrt{\lambda}}{d+1}\left[1-\left(1-\frac{T_{\min }^{2}}{T_{H}^{2}}\right)^{1 / 2}\right] \\
& =\frac{\sqrt{\lambda}}{d+1} \frac{T_{\min }^{2}}{T_{H}^{2}}+\mathcal{O}\left(T_{H}^{-4}\right) .
\end{aligned}
$$

It turns out that the leading term, quadratic in inverse temperature, gives the dominant contribution in the whole deconfining phase. The contributions of all the other terms can only be seen around $T_{c}$. This is indeed the behavior found in the lattice simulation for pure gauge theory with gauge group $\mathrm{SU}(3), \mathrm{SU}(4)$ and $\mathrm{SU}(5)[19,20]$, and even in QCD with unquenched quarks [19]. The lattice data can be well fitted by the formula

$$
-2 \log \mathcal{L}^{R}=a+b\left(\frac{T_{c}}{T}\right)^{2} .
$$


The values of $a$ and $b$ change with the gauge groups, and for $\mathrm{SU}(3), \mathrm{SU}(4)$ and $\mathrm{SU}(5)$ are roughly in the following intervals [19, 20,44]

$$
a \sim-(0.1-0.3), \quad b \sim 1.1-1.8 .
$$

As in the fuzzy bag model, one may estimate the values of $a$ and $b$ if the fit is supposed to be valid in the whole deconfining region. In the high temperature limit $\mathcal{L}^{R} \rightarrow 1$, which forces $a$ to vanish. Close to $T_{c}$, the renormalized Polyakov loop is found to approach $1 / 2$ for different gauge groups [20]. This fixes $b \approx 2 \log 2 \approx 1.39$, which is indeed in the above region.

With the expressions (2.7), (2.8), one obtains from (4.9)

$$
a=0, \quad b=\frac{d-1}{d^{2}} \sqrt{\lambda} .
$$

For $d=3$, the above lattice result for $b$ then corresponds to the 't Hooft coupling interval

$$
\lambda \sim 24-66
$$

One finds that the coupling $\lambda$ is indeed large. In other words, it is possible to reproduce the lattice result for $b$ with a reasonable coupling in a self-consistent way. This can be compared with the calculation of the jet quenching parameter using gauge/string duality [45]. Choosing $\lambda=6 \pi$, the result for the jet quenching parameter is in agreement with that determined from RHIC data. One may also confirm this with the value of $\mathcal{L}^{R}$ at $T_{c}$. From (4.8) one finds

$$
\mathcal{L}^{R}\left(T_{c}\right) \approx \exp \left[-\frac{d-1}{d(d+1)} \sqrt{\lambda}\right] .
$$

Requiring $\mathcal{L}^{R}\left(T_{c}\right) \approx 1 / 2$ for $d=3$ gives

$$
\lambda \approx 36 \log ^{2} 2 \approx 17.3, \quad b \approx 4 / 3 \log 2 \approx 0.9 .
$$

The result for the 't Hooft coupling $\lambda$ is a little smaller than that by directly fitting the parameter $b$. This is of course due to the deviation of our prediction for $a$. In figure 3 we plot our result for the renormalized Polyakov loop with such a 't Hooft coupling, and compare it to the lattice result for pure $\mathrm{SU}(3)$ gauge theory [43]. Notice that the early data here shows the Polyakov loop is slightly smaller than $1 / 2$ at $T_{c}$. Above $3 T_{c}$ the deviation between the two curves becomes large, where the lattice result overshoots the asymptotic value. Again this is due to the difference between our prediction and the lattice fit (4.10) for the parameter $a$. Introducing a nonzero value of $a$ as in [22] by hand reduces the deviation.

\section{Results for lower spacetime dimensions}

The lattice data in $2+1$ dimension shows that the trace anomaly, normalized in unit of $T^{3}$, is dominated by a linear term in inverse temperature [25, 46]. This could be compared with the behavior of the quark potential at zero temperature. We know in this case the 


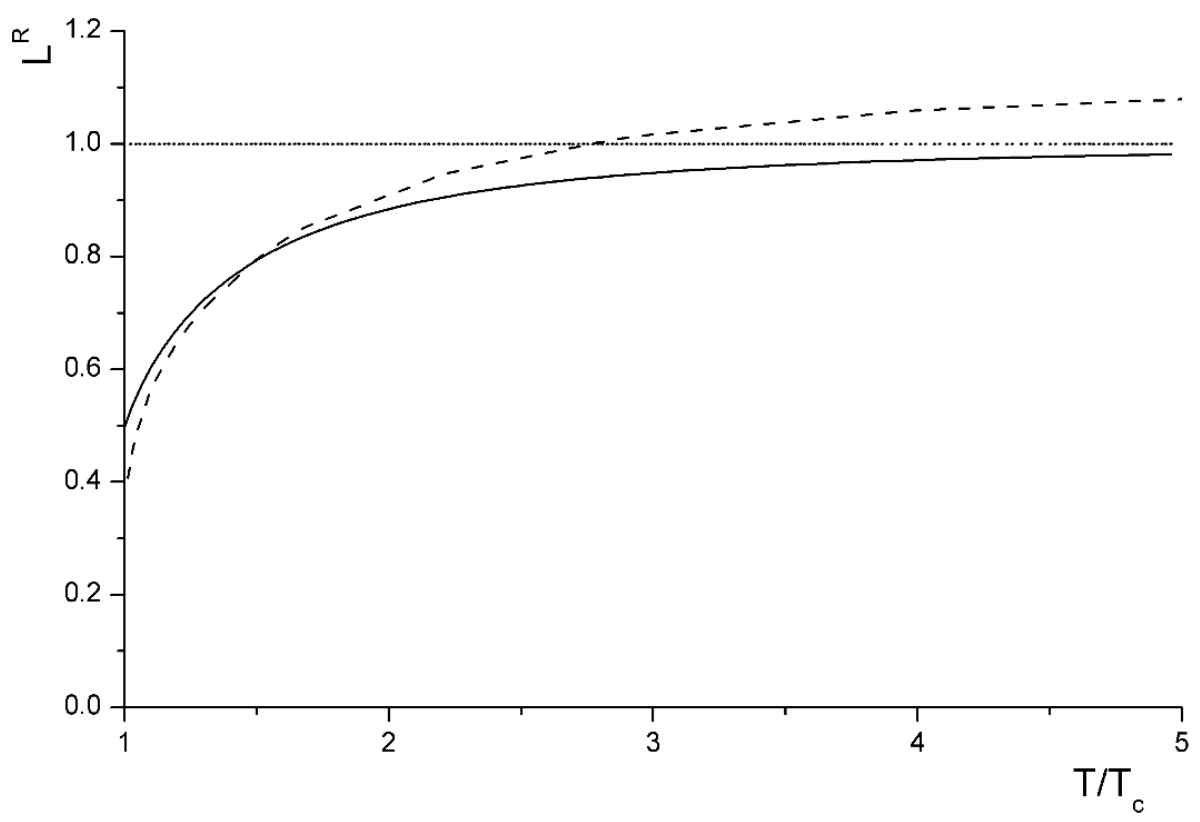

Figure 3. Holographic result (4.8) with $d=3$ and $\lambda$ given in (4.15) for the renormalized Polyakov loop versus $T / T_{c}$ (solid line), in comparison to the lattice data for pure $\mathrm{SU}(3)$ gauge theory (dashed line) [43]. The asymptotic value $\mathcal{L}^{R}=1$ is also plotted (dotted line).

perturbative potential is logarithmic, and the linear confining potential, if existing, would be roughly a linear correction at short distance. From the holographic calculation in the gravity-dilaton system such a term could be mimicked by fine-tuning a parameter which controls the order of deconfinement transition and scaling of hadron spectra [25]. However, explicit calculation shows that the power of the dominant contribution should be related to the dimension of the operator dual to the dilaton field [26]. It is also proposed that such a term could be completely due to the leading perturbative corrections [47].

Our present calculation shows that for any space dimension $d \geq 1$, the quadratic term in inverse temperature makes the dominant contribution to $\Delta / T^{d}$. The contradiction with the lattice result indicates that the Hawking-Page transition is not proper to describe deconfinement in $2+1$ dimension. This can be anticipated, since we do not know whether the duality can be generalized to $d \neq 3$ or not. As for the Polyakov loop, the quadratic term is vanishing when $d=1$. This could be compared with the fact that in $1+1$ dimension the pertubative potential by itself is linear. In $2+1$ dimension, eq. (4.12) seems to indicate the existence of a nonzero quadratic term. Again, this may not be the true situation, due to the same reasoning as the trace anomaly.

\section{Discussion}

We have shown that the quadratic thermal terms of gauge theory observed in lattice data can be generated naturally on the gravity side. The reason can be traced back to the difference of the bulk metric when the boundary space is compact or not. If one naively compares metric (2.2) with (2.10), one finds that in (2.2) there is an additional constant term in the 
warp factor. The same pattern can also be found comparing (2.3) with (2.9). According to the dictionary of the duality $[4,5]$, such a term would correspond to a dimension-2 operator at the boundary, or a tachyonic field in the bulk. In our calculation no such field is introduced, and the correction is completely due to change of the boundary topology. The comparison between the thermal properties of the theory on $S^{3}$ and $R^{3}$ also shows that such contributions appear together with the phase transition. Therefore they could the embers of confinement above $T_{c}$, i.e., the remaining effects of the linear potential in the deconfined phase. Combining the confinement argument in [8] through the Wilson loop, one may suspect that these quadratic terms are also due to global change of the bulk spacetime, rather than some local effects.

The price to pay for such contributions, and confinement, in the present case is the compactness of the boundary space. Therefore the properties found here may not be directly related to those in flat space time. However, the idea behind the model could be employed to describe realistic gauge theory. As we have explained, the shape of all the quantities in the model can be obtained by combining the behavior at $T_{\min }$ and in the high temperature limit. Therefore, the results presented here could be universal among theories with a minimum temperature of the black hole phase. The numerous studies in the gravity-dilaton system have partially confirmed this. In particular, it is pointed out that the existence of a minimum temperature of the black hole solution is closely related to confinement at zero temperature $[38,39]$. If this is in general true, the results shown here may be extended to all confining theories.

\section{Acknowledgments}

F.Z. thanks Pietro Colangelo, Floriana Giannuzzi and Stefano Nicotri for helpful discussions. This work is partially supported by the National Natural Science Foundation of China under Grant No. 11445001 and No. 11135011.

Open Access. This article is distributed under the terms of the Creative Commons Attribution License (CC-BY 4.0), which permits any use, distribution and reproduction in any medium, provided the original author(s) and source are credited.

\section{References}

[1] R. Akhoury and V.I. Zakharov, On nonperturbative corrections to the potential for heavy quarks, Phys. Lett. B 438 (1998) 165 [hep-ph/9710487] [INSPIRE].

[2] K.G. Chetyrkin, S. Narison and V.I. Zakharov, Short distance tachyonic gluon mass and 1/Q $Q^{2}$ corrections, Nucl. Phys. B 550 (1999) 353 [hep-ph/9811275] [INSPIRE].

[3] J.M. Maldacena, The Large- $N$ limit of superconformal field theories and supergravity, Adv. Theor. Math. Phys. 2 (1998) 231 [Int. J. Theor. Phys. 38 (1999) 1113] [hep-th/9711200] [INSPIRE].

[4] S.S. Gubser, I.R. Klebanov and A.M. Polyakov, Gauge theory correlators from noncritical string theory, Phys. Lett. B 428 (1998) 105 [hep-th/9802109] [InSPIRE]. 
[5] E. Witten, Anti-de Sitter space and holography, Adv. Theor. Math. Phys. 2 (1998) 253 [hep-th/9802150] [INSPIRE].

[6] J.M. Maldacena, Wilson loops in large-N field theories, Phys. Rev. Lett. 80 (1998) 4859 [hep-th/9803002] [INSPIRE].

[7] S.-J. Rey and J.-T. Yee, Macroscopic strings as heavy quarks in large- $N$ gauge theory and anti-de Sitter supergravity, Eur. Phys. J. C 22 (2001) 379 [hep-th/9803001] [InSPIRE].

[8] E. Witten, Anti-de Sitter space, thermal phase transition and confinement in gauge theories, Adv. Theor. Math. Phys. 2 (1998) 505 [hep-th/9803131] [INSPIRE].

[9] O. Andreev and V.I. Zakharov, Heavy-quark potentials and AdS/QCD, Phys. Rev. D 74 (2006) 025023 [hep-ph/0604204] [INSPIRE].

[10] F. Zuo and T. Huang, Comments on the two-dimensional power correction in the soft wall model, Chin. Phys. Lett. 25 (2008) 3601 [arXiv:0801.1172] [InSPIRE].

[11] P.N. Meisinger, T.R. Miller and M.C. Ogilvie, Phenomenological equations of state for the quark gluon plasma, Phys. Rev. D 65 (2002) 034009 [hep-ph/0108009] [INSPIRE].

[12] G. Boyd et al., Thermodynamics of SU(3) lattice gauge theory, Nucl. Phys. B 469 (1996) 419 [hep-lat/9602007] [INSPIRE].

[13] R.D. Pisarski, Fuzzy Bags and Wilson Lines, Prog. Theor. Phys. Suppl. 168 (2007) 276 [hep-ph/0612191] [INSPIRE].

[14] P. Alba et al., Polyakov loop and gluon quasiparticles: a self-consistent approach to Yang-Mills thermodynamics, arXiv: 1402.6213 [INSPIRE].

[15] M. Panero, Thermodynamics of the QCD plasma and the large-N limit, Phys. Rev. Lett. 103 (2009) 232001 [arXiv:0907.3719] [INSPIRE].

[16] A.M. Polyakov, Thermal Properties of Gauge Fields and Quark Liberation, Phys. Lett. B 72 (1978) 477 [INSPIRE].

[17] G. 't Hooft, On the Phase Transition Towards Permanent Quark Confinement, Nucl. Phys. B 138 (1978) 1 [INSPIRE].

[18] O. Kaczmarek, F. Karsch, P. Petreczky and F. Zantow, Heavy quark anti-quark free energy and the renormalized Polyakov loop, Phys. Lett. B 543 (2002) 41 [hep-lat/0207002] [INSPIRE].

[19] E. Megias, E. Ruiz Arriola and L.L. Salcedo, Dimension two condensates and the Polyakov loop above the deconfinement phase transition, JHEP 01 (2006) 073 [hep-ph/0505215] [INSPIRE].

[20] A. Mykkanen, M. Panero and K. Rummukainen, Casimir scaling and renormalization of Polyakov loops in large- $N$ gauge theories, JHEP 05 (2012) 069 [arXiv:1202.2762] [INSPIRE].

[21] O. Andreev, Some Thermodynamic Aspects of Pure Glue, Fuzzy Bags and Gauge/String Duality, Phys. Rev. D 76 (2007) 087702 [arXiv:0706.3120] [INSPIRE].

[22] O. Andreev, Renormalized Polyakov Loop in the Deconfined Phase of SU(N) Gauge Theory and Gauge/String Duality, Phys. Rev. Lett. 102 (2009) 212001 [arXiv:0903.4375] [INSPIRE].

[23] A. Karch, E. Katz, D.T. Son and M.A. Stephanov, Linear confinement and AdS/QCD, Phys. Rev. D 74 (2006) 015005 [hep-ph/0602229] [INSPIRE]. 
[24] O. Andreev, 1/Q $Q^{2}$ corrections and gauge/string duality, Phys. Rev. D 73 (2006) 107901 [hep-th/0603170] [INSPIRE].

[25] M. Caselle et al., Thermodynamics of SU(N) Yang-Mills theories in 2+1 dimensions II. The Deconfined phase, JHEP 05 (2012) 135 [arXiv:1111.0580] [INSPIRE].

[26] F. Zuo, Thermal power terms in the Einstein-dilaton system, JHEP 06 (2014) 143 [arXiv: 1404.4512] [INSPIRE].

[27] A. Dumitru, Y. Guo, Y. Hidaka, C.P.K. Altes and R.D. Pisarski, Effective Matrix Model for Deconfinement in Pure Gauge Theories, Phys. Rev. D 86 (2012) 105017 [arXiv:1205.0137] [INSPIRE].

[28] S.W. Hawking and D.N. Page, Thermodynamics of Black Holes in anti-De Sitter Space, Commun. Math. Phys. 87 (1983) 577 [InSPIRE].

[29] S.S. Gubser, I.R. Klebanov and A.W. Peet, Entropy and temperature of black 3-branes, Phys. Rev. D 54 (1996) 3915 [hep-th/9602135] [INSPIRE].

[30] J. Casalderrey-Solana, H. Liu, D. Mateos, K. Rajagopal and U.A. Wiedemann, Gauge/String Duality, Hot QCD and Heavy Ion Collisions, arXiv:1101.0618 [INSPIRE].

[31] W. Fischler and S. Kundu, Strongly Coupled Gauge Theories: High and Low Temperature Behavior of Non-local Observables, JHEP 05 (2013) 098 [arXiv: 1212.2643] [INSPIRE].

[32] D. Li, J. Liao and M. Huang, Enhancement of jet quenching around phase transition: result from the dynamical holographic model, Phys. Rev. D 89 (2014) 126006 [arXiv:1401.2035] [INSPIRE].

[33] S.S. Gubser, A. Nellore, S.S. Pufu and F.D. Rocha, Thermodynamics and bulk viscosity of approximate black hole duals to finite temperature quantum chromodynamics, Phys. Rev. Lett. 101 (2008) 131601 [arXiv:0804.1950] [inSPIRE].

[34] S.S. Gubser, S.S. Pufu and F.D. Rocha, Bulk viscosity of strongly coupled plasmas with holographic duals, JHEP 08 (2008) 085 [arXiv:0806.0407] [INSPIRE].

[35] U. Gürsoy, E. Kiritsis, G. Michalogiorgakis and F. Nitti, Thermal Transport and Drag Force in Improved Holographic QCD, JHEP 12 (2009) 056 [arXiv:0906.1890] [INSPIRE].

[36] G. Policastro, D.T. Son and A.O. Starinets, The Shear viscosity of strongly coupled $N=4$ supersymmetric Yang-Mills plasma, Phys. Rev. Lett. 87 (2001) 081601 [hep-th/0104066] [INSPIRE].

[37] S.S. Gubser and A. Nellore, Mimicking the QCD equation of state with a dual black hole, Phys. Rev. D 78 (2008) 086007 [arXiv: 0804.0434] [INSPIRE].

[38] U. Gürsoy, E. Kiritsis, L. Mazzanti and F. Nitti, Deconfinement and Gluon Plasma Dynamics in Improved Holographic QCD, Phys. Rev. Lett. 101 (2008) 181601 [arXiv:0804.0899] [INSPIRE].

[39] U. Gürsoy, E. Kiritsis, L. Mazzanti and F. Nitti, Holography and Thermodynamics of $5 D$ Dilaton-gravity, JHEP 05 (2009) 033 [arXiv:0812.0792] [INSPIRE].

[40] J. Noronha, Connecting Polyakov Loops to the Thermodynamics of $S U\left(N_{c}\right)$ Gauge Theories Using the Gauge-String Duality, Phys. Rev. D 81 (2010) 045011 [arXiv:0910.1261] [INSPIRE].

[41] J. Noronha, The Heavy Quark Free Energy in QCD and in Gauge Theories with Gravity Duals, Phys. Rev. D 82 (2010) 065016 [arXiv:1003.0914] [INSPIRE]. 
[42] A. Bazavov et al., Equation of state and QCD transition at finite temperature, Phys. Rev. D 80 (2009) 014504 [arXiv: 0903.4379] [InSPIRE].

[43] S. Gupta, K. Huebner and O. Kaczmarek, Renormalized Polyakov loops in many representations, Phys. Rev. D 77 (2008) 034503 [arXiv:0711.2251] [inSPIRE].

[44] E. Megias, E. Ruiz Arriola and L.L. Salcedo, The Quark-antiquark potential at finite temperature and the dimension two gluon condensate, Phys. Rev. D 75 (2007) 105019 [hep-ph/0702055] [INSPIRE].

[45] H. Liu, K. Rajagopal and U.A. Wiedemann, Calculating the jet quenching parameter from AdS/CFT, Phys. Rev. Lett. 97 (2006) 182301 [hep-ph/0605178] [INSPIRE].

[46] P. Bialas, L. Daniel, A. Morel and B. Petersson, Thermodynamics of SU(3) Gauge Theory in 2+ 1 Dimensions, Nucl. Phys. B 807 (2009) 547 [arXiv:0807.0855] [InSPIRE].

[47] P. Bicudo, R.D. Pisarski and E. Seel, Matrix model for deconfinement in a $S U\left(N_{c}\right)$ gauge theory in 2+1 dimensions, Phys. Rev. D 89 (2014) 085020 [arXiv:1402.5137] [INSPIRE]. 